\title{
An evaluation of a new test of reactive agility and its relationship to sprint speed and change of direction speed
}

\author{
J.M. Sheppard ${ }^{a, b, c, *}$, W.B. Young ${ }^{b}$, T.L.A. Doyle ${ }^{c}$, \\ T.A. Sheppard ${ }^{\text {b,c }}$, R.U. Newton ${ }^{c}$ \\ ${ }^{a}$ Australian Institute of Sport, Belconnen, Australia \\ b School of Human Movement and Sport Sciences, University of Ballarat, Australia \\ ' School of Biomedical and Sport Sciences, Edith Cowan University, Australia
}

* Corresponding author.

E-mail address: jeremy.sheppard@ausport.gov.au (J.M. Sheppard).

\section{Introduction}

Agility is an essential component in most field and team sports. Traditional definitions of agility have simply identified speed in directional changes as the defining component. ${ }^{1}$ Young et al. ${ }^{2}$ identified agility as comprising two key sub-components; speed in 
changing direction, and cognitive factors. ${ }^{2}$ More recently, agility has been identified as "a rapid whole body movement with change of velocity or direction in response to a stimulus". ${ }^{3}$ This definition recognises the inclusion of cognitive skills in determining agility performance, and this definition applies to open skills only. Open skills cannot be pre-planned, whereas closed skills, such as sprint running or pre-determined changes of direction, can be pre-planned. ${ }^{4}$

In many sports, such as football codes, athletes are required to accelerate, decelerate and change direction throughout the game. ${ }^{5}$ Often these movements are in response to cues such as the movements of a ball, or the actions of opposition players. Considering that cognitive components are an integral part of sports that require a reaction to a stimulus, and that there are differences between players in the ability to "read and react" to these sport-specific cues, ${ }^{6,7}$ it would appear ideal to evaluate athletes using a test of agility that includes a reaction to a stimulus that is similar to that of the sport.

Zig-zag running speed tests have classically been used to assess "agility". These tests have evolved from highly generic movement patterns, ${ }^{8}$ to those which may better mimic the demands of a sport or sports. ${ }^{1}$ However, these tests are closed skill tests, as all of the movements can be pre-planned, and there is no response to a stimulus. The term change of direction speed (CODS) has been used to describe these movements, in order to distinguish between CODS and the current definition of agility. ${ }^{2}$

In order to address the need for an evaluation of unplanned, open skill movements, several researchers have implemented tests that require a change of direction in response to a generic cue, such as a light bulb or computerised direction indicator. ${ }^{9-11}$ However, the efficacy of these generic cues with athletes has been questioned. $3,6,12$

Specifically, it has been questioned whether or not the use of generic cues is appropriate to evaluate an athlete's skill level, because perceptual expertise is linked not only to increased visual search rates but also specific search cues and accuracy of domain-specific responses. ${ }^{6,13,14}$ Therefore, it is unlikely that generic cue reaction tests are valid in discriminating between higher and lower performers in a particular sport. In support of this, studies that have shown differences between higher and lower performers in anticipation, decisionmaking speed and decision-making accuracy, have done so with sport-specific stimuli. ${ }^{6,7,13}$

Another consideration that supports the need for highly specific cues, rather than light bulbs or direction indicators, is the element of anticipation as part of the entire cognitive process involved in reacting to a sport-specific stimulus. When using a generic cue, such as a light bulb that is either "off" or "on", there is no opportunity for an athlete to anticipate the direction or timing of this stimulus. However, in a sporting environment, athletes who have anticipatory expertise are able recognise and attend to different cues that occur earlier in the presentation of a stimulus. ${ }^{6,14}$

A practical example of this would be that of defensive play in a football code sport. A defender who has developed cognitive expertise that is relevant to that skill will attend to cues earlier in the movement of the offender, when compared to the performance that would be demonstrated by a novice defender. The novice defender may require the entire skill to be executed (e.g., cross-over step and direction change) before making the correct decision and responding to the cue. The expert defender is likely to recognise earlier cues such as torso position, foot placement or other unique cues in order to make the correct decision earlier in the execution of the skill. A generic stimulus, although able to measure response-time, is not able to include visual search and allow the athlete to demonstrate any expertise in movement pattern recognition.

It has been suggested that, although these perceptual-cognitive considerations are likely needed to be included in a true agility test, the aim of most "agility" tests is actually to test only CODS. ${ }^{15}$ Indeed, CODS tests that generally involve sprints and changes of direction around stationary objects, are commonly used in sport settings. ${ }^{1,8}$ In addition to the lack of cognitive demands of closed skill testing methods, research evidence (and coaching observations) suggest that closed skill changes of direction impose significantly different stresses on the body in comparison to open skill movements. ${ }^{9}$

No literature was found that evaluated a sportspecific, physical performance test of agility that included anticipation and decision-making using a three-dimensional stimulus. A field test that involved a sport-specific stimulus to which the athletes must "read and react", would appear ideal for the measurement of agility. Therefore, the purpose of this research study was to develop and evaluate a new test of agility for football codes that involved perceptual, decision-making and movement response components (sprint running and direction change). The evaluation included measures of test-retest reliability and inter-rater reliability. Validity was assessed by comparing two groups of Australian football players of differing 
competition level, and performing a correlation analysis between a straight sprinting test, planned change of direction speed test and the agility test.

\section{Methods}

\section{Participants}

Thirty-eight Australian football players, with a mean \pm S.D. age, height and mass of $21.8 \pm 3.2 \mathrm{y}$, $181.7 \pm 7.5 \mathrm{~cm}$, and $82.0 \pm 9.9 \mathrm{~kg}$, respectively, were involved in this study. All players were involved in the same training regimen with respect to training variables such as volume and intensity. The players were classified into two groups.

\section{Higher performance group (HPG) $(n=23)$}

Composed of senior league Western Australian football league (WAFL) players, with a mean \pm S.D. age, height and mass of $23.1 \pm 3.7 \mathrm{y}, 181.9 \pm 6.7 \mathrm{~cm}$, and $80.6 \pm 8.3 \mathrm{~kg}$, respectively. To be eligible for the HPG, players were registered with a state (WAFL) team at the time of the study. In addition, the players in the HPG had previous experience (i.e., the previous season) in the WAFL. Four of these players had played previously for a national league team, i.e., Australian football league (AFL) level.

Lower performance group (LPG) $(n=14)$

Composed of reserve grade players from the same team competing in the WAFL, with a mean \pm S.D. age, height and mass of $19.9 \pm 1.5 \mathrm{y}$, $183.3 \pm 7.6 \mathrm{~cm}$, and $84.1 \pm 11.9 \mathrm{~kg}$, respectively. These participants had previous playing experience in the WAFL reserves, but not in the WAFL senior league, and were deemed most likely to play at the reserve grade level for the full season.

\section{Protocols}

After the initial familiarisation session, testing took place over two sessions for the LPG in order to assess test-retest reliability and inter-rater reliability. These occasions were separated by $48 \mathrm{~h}$. For the comparison between the higher and lower performance levels, the testing of the HPG took place on one occasion, 1 week prior to the testing of the LPG, $48 \mathrm{~h}$ after the initial familiarisation session. The testing procedure and time of day was identical for all participants. In addition, the 20 min warm-up procedure was kept constant for all sessions; involving a general running warm-up, basic Australian football drills and sub-maximal effort trials of the tests. No training took place $24 \mathrm{~h}$ prior to testing for any group to minimise the effects of fatigue on test results.

Testing took place on a wooden, indoor floor surface to control for environmental conditions such as temperature, wind and ground conditions. Players were instructed to wear the same footwear for all sessions. Test results were collected using the kinematic measurement system (KMS) infrared timing light system interfaced with compatible computer software (Fitness Technologies, Adelaide, Australia).

\section{Straight sprint (10 mSS)}

Athletes were tested on a $10 \mathrm{~m}$ straight sprint. The start commenced from a standing position, with the chest just behind the infra-red timing beam so that any forward movement by the athlete triggered the timer to begin. The athletes were instructed to begin with their preferred foot forward, with their front toe placed on a line marked on the floor. Instructions were given so that the players did not move backward prior to initiating the sprint, and any attempts that involved a backward rocking motion before forward movement were discarded and re-trialled. The recorded score for this test was the mean of two trials.

\section{Change of direction speed test (CODST)}

Athletes underwent a sprint test of approximately 8-9 $\mathrm{m}$ (depending on the nature and angle by which the athlete performed the change of direction), with a single direction change, performing two trials to each direction (Fig. 1). These dimensions were chosen so that the distance covered was similar to the reactive agility test (RAT) and with a direction change and distance that is representative of the change that occurs in the RAT test.

The dimensions of the CODS test were determined by running pilot trials of the RAT prior to data collection. Using six players of similar skill to those involved in the research study, trials of the RAT were run for the purposes of estimating the point where the subjects typically initiated a directional

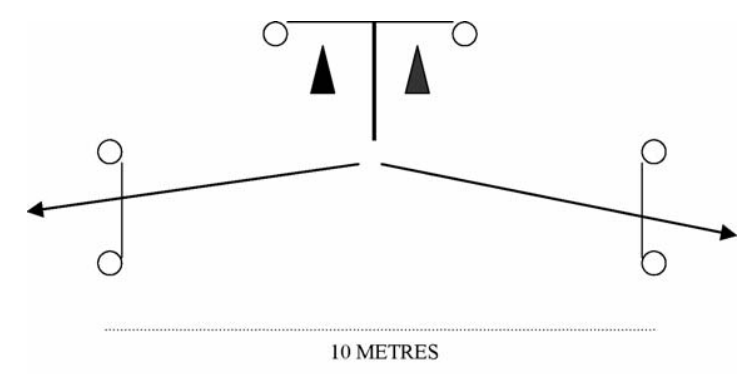

Figure 1 Change of direction speed test (CODST). 
change. Calculating an exact mean during the pilot testing was not possible due to the varied nature in which the participants changed direction. Some participants adopted a distinct "cutting" action, and this point could be measured. However, due to the unplanned directional change required of the RAT, some participants adopted a less distinct, rounded directional change, making calculations of a distinct distance at which the directional change occurred impossible. The distance chosen, at $1.5 \mathrm{~m}$ forward, is where the obstacle was placed in the CODST. The recorded score for this test was the mean of all four trials, which consisted of two trials to the left and two trials to the right, as no significant difference existed between left and right direction change trials.

Timing gates were placed at the start position and finish positions for the CODST. Similar to the $10 \mathrm{mSS}$ test procedure, the athletes were instructed to begin with their preferred foot on the marked line, with the chest just behind the infra-red beam of the timing gates. The athletes were instructed to avoid moving backwards prior to initiating forward movement.

\section{Reactive agility test (RAT)}

The athlete began on the marked line, as illustrated in Fig. 2. Timing gates were placed $5 \mathrm{~m}$ to the left and right, $2 \mathrm{~m}$ forward of the start line. Therefore, the timing gates were placed $10 \mathrm{~m}$ from each other.

The tester (researcher) stood opposite, and facing, the participants. The tester stood on a timing mat that was inter-faced with the computing software used with this timing gate system. In this system set-up, when the tester moved off of the timing mat, an audible beep was emitted and the timing began.

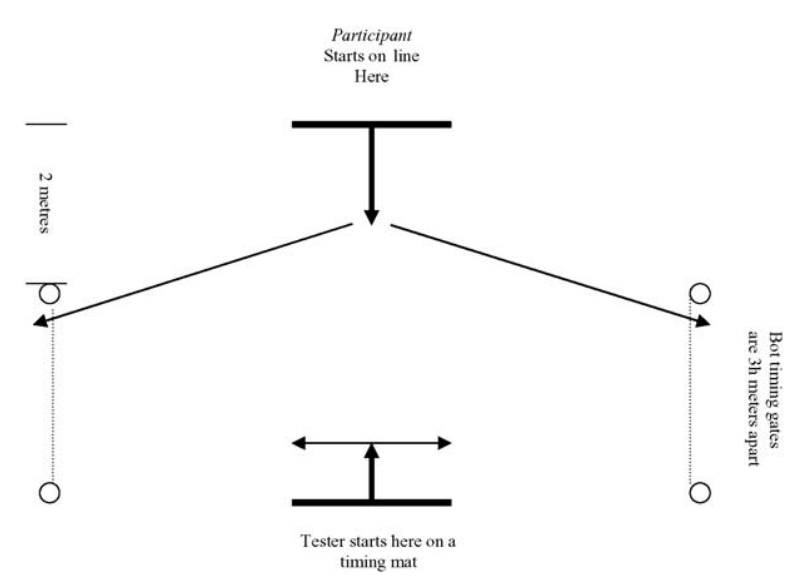

Figure 2 Reactive agility test (RAT).

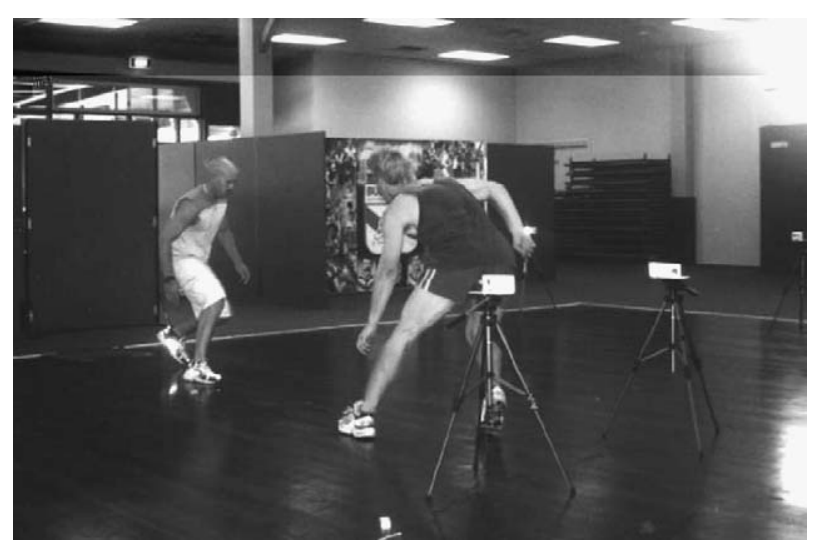

Figure 3 The reactive agility test stimulus.

Each test trial involved the tester initiating movement, and thereby beginning the timing. The athlete reacted to the movements of the tester, forward, then to the left or right in response to, and in the same direction as, the left or right movement of the tester (Fig. 3). The timing stopped when the athlete triggered the timing beam on either side.

The tester displayed one of four possible scenarios for the athlete to react to, however the athletes did not explicitly know this. The four possible scenarios all involved steps of approximately $0.5 \mathrm{~m}$ and were presented in a random order that was different for each athlete:

1. Step forward with right foot and change direction to the left.

2. Step forward with the left foot and change direction to the right.

3. Step forward with the right foot, then left, and change direction to the right.

4. Step forward with the left foot, then right, and change direction to the left.

There was an equal amount of each scenario for each participant. The test protocol involved randomised presentation of 4 different cues, for a total of 12 trials. These cues created varying demands on the participants (as in a game setting), resulting in inter-trial variability. For this reason, the recorded score used was the mean of all trials (12), which was an average of all trials to the left (6) and to the right (6).

The participants sprinted forward prior to any change of direction, in reaction to the forward movement of the tester. The participant was instructed to recognise the cues as soon as possible (essentially while moving forward) and react by changing direction and sprinting through the gates on the left or right in response. The 
participants were instructed to emphasise accuracy (decision-making accuracy) and speed of movement.

The primary researcher of this investigation presented the stimulus to the LPG, as well as the HPG. For inter-rater reliability comparisons, an assistant researcher, of similar physical capabilities, was recruited (tester B).

To increase consistency between subjects and between testers, the two testers followed these instructions for the test:

1. Testers took approximately $0.5 \mathrm{~m}$ steps forward (for the first steps prior to changing direction), before turning to the left or right, always using the outside leg as the driving leg. (Right leg push off for a change of direction to the left.)

2. The researchers allowed no one access to the information on the directional changes and, if queried on further specifics of the possible scenarios, the pre-planned movements of the tester were described as follows:

"The stimulus (change of direction) will be presented soon after the tester moves forward."

3. The researchers did not discuss with the participants the likelihood of whether or not equal trials would be given for each direction, nor did they discuss desired outcomes. The only information given to the participants was that which was related to testing instructions and procedures (i.e., number of trials, protocols).

4. The testers wore the same footwear and clothing for all testing sessions and the test was performed in an indoor gymnasium to control for conditions. The testers wore running shoes and athletic training clothing.

For all testing occasions, the testing was conducted in this order: SS, CODS and finally the RAT.

\section{Statistical analysis}

Test-retest reliability was examined using ICC, change in the mean, technical error of measure- ment (TEM) as well as applying a paired samples $t$-test to determine whether or not there was a significant difference between the two testing sessions. Inter-rater reliability was examined by using a paired samples $t$-test for comparison of the results obtained by tester A on day 1 , and tester B on day 2 .

\section{Validity}

Potential group differences between the LPG and the HPG were assessed using ANOVA. Pearson correlations were used on the entire participant pool to assess commonality between the $10 \mathrm{mSS}$, CODST and RAT.

Ninety-five percent confidence intervals were applied where appropriate.

\section{Results}

\section{Reliability}

The means \pm S.D., ICCs and TEMs for the three tests over the two testing sessions are shown in Table 1. Paired sample $t$-tests revealed no significant $(p<0.05)$ differences between the testing occasions.

\section{Inter-rater reliability of the reactive agility test}

No significant differences $(p<0.05)$ were observed between the RAT scores that were obtained by tester A, in comparison with the scores obtained by tester B. As a further measure, ICC (alpha) analysis revealed a high level of reliability between tester $A$ and tester $B(r=0.904)$.

\section{Validity}

The mean scores \pm S.D.s and effect size for the LPG and HPG for all three tests are shown in Table 2. ANOVA revealed no significant differences between the HPG and the LPG in the $10 \mathrm{mSS}$ and CODS tests. However, the HPG was faster in performance of the

Table 1 Mean \pm S.D., intra-class correlation (ICC) and technical error of measurement (TEM) of test-retest results of the straight sprint, change of direction speed test, and reactive agility test (s)

\begin{tabular}{lllll}
\hline Test & Day 1 & Day 2 & ICC & TEM \\
\hline Straight sprint (s) & $1.892 \pm 0.050$ & $1.923 \pm 0.060$ & 0.865 & 0.006 \\
Change of direction speed test (s) & $1.612 \pm 0.100$ & $1.606 \pm 0.080$ & 0.865 & 0.046 \\
Reactive agility test (s) & $1.637 \pm 0.080$ & $1.635 \pm 0.090$ & 0.878 & 0.005 \\
\hline
\end{tabular}


Table 2 Mean difference between lower performance group and higher performance group on the speed variables

\begin{tabular}{lcc}
\hline Variable & Lower performance group $(n: 15)$ & High performance group $(n: 23)$ \\
\hline 10 m straight sprint $(s)$ & 1.895 & $1.909(0.7 \%$ slower $)$ \\
Standard deviation & \pm 0.053 & \pm 0.067 \\
Effect size statistic & & 0.23 \\
Change of direction speed (s) & 1.609 & $1.640(1.9 \%$ slower $)$ \\
Standard deviation & \pm 0.092 & \pm 0.086 \\
Effect size statistic & & 0.35 \\
Reactive agility test $(s)$ & 1.639 & $1.553(5.2 \% \text { faster })^{*}$ \\
Standard deviation & \pm 0.082 & \pm 0.070 \\
Effect size statistic & & 1.13 \\
\hline
\end{tabular}

"Statistical significance $(p=<0.05)$.

Table 3 Correlations between the speed, change of direction, and agility variables

\begin{tabular}{lll}
\hline & Change of direction speed test & Reactive agility test \\
\hline Straight sprint test & $0.738^{* *}(54 \%)$ & $0.333^{*}(11 \%)$ \\
Change of direction speed test & & $0.321^{*}(10 \%)$ \\
\hline
\end{tabular}

Percent common variance is shown in brackets for selected comparisons.

" Significant at the 0.05 level.

** Significant at the 0.01 level.

agility measure (RAT) and this difference was statistically significant $(p=0.001)$.

The three correlation analyses conducted between the $10 \mathrm{mSS}$, CODS and RAT were all statistically significantly $(p<0.05)$. These results are presented in Table 3.

\section{Discussion}

Although the test-retest ICC value for the RAT found in this investigation was lower than some previous research studies involving tests of planned direction changes, ${ }^{16,17}$ the ICC of 0.878 for the RAT is higher than other studies that measured the reliability of tests that involved unplanned direction changes. ${ }^{7,10,11}$. Importantly, all of the tests investigated produced ICC values that are acceptably reliable $(>0.80)$ for physical performance tests. ${ }^{18}$

Inter-rater reliability was assessed using two statistical methods. Firstly, a dependent samples $t$-test determined that no significant differences existed between the results obtained by tester A, and the results of tester $B$. In addition, an average measure ICC was computed between the scores of tester $B$ and the scores from the primary tester. The ICC score was 0.904 for comparisons between tester
$A$ and tester $B$. The lack of a significant difference between the test results, along with the high ICC value, indicates that the RAT is not only acceptably test-retest reliable, but also inter-rater reliable.

The HPG produced significantly $(p=0.001)$ superior scores in the performance of the RAT in comparison with the LPG, with effect size calculations further demonstrating the significance of this difference (Table 2). However, there were no other significant differences between the groups on any of the other dependent variables. In fact, the LPG had non-significant, but superior scores on the $10 \mathrm{mSS}$ and the CODST. This result suggests that traditional closed skill sprint and sprints with direction change tests may not adequately distinguish between players of different levels of competition football.

It could be suggested that the difference in scores on the RAT between the groups of higher and lower performance in the present study can be explained by differences in the cognitive abilities of the athletes. If we accept that agility comprises specific physical and cognitive components, and there were no differences observed between the groups in the $10 \mathrm{mSS}$ and CODST, then this may suggest that the observed difference between the groups on the RAT was related to cognitive abilities. 
Qualitative observations during data collection indicated that many of the HPG athletes were able to "read and react" to the stimulus given, prior to the completion of the movements that comprised the entire stimulus. In other words, many athletes who were in the HPG were seemingly able complete the decision-making process at an earlier time during the stimulus presentation. This observation is in agreement with investigations that have addressed the differences between the domainspecific anticipation and decision-making skills of novices and experts in sport. 6,7,13 However, in the present study, decision-making time was not measured directly, and therefore assumptions of superior cognitive abilities of the HPG cannot be made with certainty.

The low common variance between the open skill test (RAT) and the closed skill speed tests $(10 \mathrm{mSS}$, CODST) further supports the validity of the RAT. As was observed in the present research, some low and moderate relationships have been observed between straight sprinting and sprints with directional change. ${ }^{1,19,20}$ However, when comparing the CODST and the RAT, whose physical dimensions (and demands) were identical, the common variance among these two tests was only $10 \%$, suggesting that these two tests are measuring distinct qualities.

\section{Conclusions}

This is the first study to demonstrate the importance of including a sport-specific stimulus in speed and directional change tests for separating players of differing playing levels in Australian football, and is in agreement with research of a similar nature in netball. ${ }^{7}$ This result demonstrates the importance of including sport-specific cue recognition in testing agility, and suggests that in training agility for Australian football, sport-specific cue recognition should be included as a part of the physical training. Examples of this would include evasions drills and pursuant games (i.e., tagging) for reactive agility rather than only performing directional changes around stationary objects.

It is hoped that this investigation may create an impetus for future research into the reliability and validity of this test when used with sports other than Australian football. Variations of this test may also allow for a broader application to other sports, particularly sports other than football codes. Finally, investigations into the sensitivity of the RAT to measure agility performance changes brought about through training, would be worthy of further investigation.

\section{Practical implications}

- Straight sprinting and change of direction sprints are unlikely to be adequate in assessing the physical and cognitive demands of field running sports.

- To assess on-field open skill agility performance validly, a test involving open skill agility with sport-specific cue recognition is preferable.

\section{Acknowledgement}

The authors would like to thank the Australian Institute of Sport Lab Standards Assistance Scheme for funding in support of this research project.

\section{References}

1. Draper JA, Lancaster MG. The 505 test: a test for agility in the horizontal plane. Aust J Sci Med Sport 1985;17(1):15-8.

2. Young WB, James R, Montgomery I. Is muscle power related to running speed with changes of direction? J Sport Med Phys Fit 2002;43:282-8.

3. Sheppard JM, Young WB. Agility literature review: classifications, training and testing. J Sport Sci 2006;24(9): 915-28.

4. Cox RH. Sport psychology: concepts and applications. 5th ed. New York: McGraw-Hill; 2002.

5. Docherty D, Wenger HA, Neary P. Time-motion analysis related to the physiological demands of rugby. J Hum Mov Stud 1988;14:269-77.

6. Abernethy B, Russell DG. Expert-novice difference in an applied selective attention task. J Sport Psych 1987;9:326-45.

7. Farrow D, Young W, Bruce L. The development of a test of reactive agility for netball: a new methodology. J Sci Med Sport 2005;8(1):40-8.

8. Semenick D. Tests and measurements: the $t$-test. Strength Cond J 1990;12(1):36-7.

9. Besier TF, Lloyd DG, Cochrane JL, et al. External loading of the knee joint during running and cutting manoeuvres. Med Sci Sport Exer 2001;33:1168-75.

10. Chelladurai P, Yuhasz M, Sipura R. The reactive agility test. Percep Motor Skill 1977;44:1319-24.

11. Hertel J, Denegar CR, Johnson PD, et al. Reliability of the cybex reactor in the assessment of an agility task. $J$ Sport Rehab 1999;8:24-31.

12. Farrow D, Chiver P, Hardingham C, et al. The effect of videobased perceptual training on the tennis return of serve. Int J Sport Psych 1998;29:231-42.

13. Tenenbaum G, Levy-Kolker N, Sade S, et al. Anticipation and confidence of decisions related to skilled performance. Int J Sport Psych 1996;27:293-307.

14. Williams AM, Davids K, Burwitz L, et al. Visual search and sports performance. Aust J Sci Med Sport 1993;25(2):55-65.

15. Ellis L, Gastin P, Lawrence $S$, et al. Protocols for the physiological assessment of team sport players. In: Gore CJ, 
editor. Physiological tests for elite athletes. Canberra: Australian Sports Commission; 2000. p. 128-44.

16. Alricsson M, Harms-Ringdahl K, Werner S. Reliability of sports related functional tests with emphasis on speed and agility in young athletes. Scand $J$ Med Sci Sport 2001;11:229-32.

17. Pandorf CE, Nindl BC, Montain SJ, et al. Reliability assessment of two military relevant occupational physical performance tests. Can J Appl Phys 2003;28(1):27-37.
18. Thomas JR, Nelson JK. Research methods in physical activity. Champaign, Illinois: Human Kinetics; 2001.

19. Pauole K, Madole K, Garhammer J, et al. Reliability and validity of the $t$-test as a measure of agility, leg power, and leg speed in college-aged men and women. J Strength Cond Res 2000;14(4):443-50.

20. Young WB, MCDowell MH, Scarlett BJ. Specificity of sprint and agility training methods. J Strength Cond Res 2001;15(3):310-5. 